\title{
Red de transición aumentada y lenguaje formal para la danza Bhāratanāṭam
}

\author{
Rosario Romero-Conde ${ }^{1}$, Miguel Murguía-Romero ${ }^{1,2}$ \\ 1 Grupo cultural Nātya Sūtra, Ciudad de México, \\ México \\ 2 UNAM, FES Iztacala, \\ México \\ miguelmurguia@ciencias.unam.mx
}

\begin{abstract}
Resumen. El Bhāratanātyam es una danza clásica de la India, ancestral, se practica en muchos países, está documentada en textos antiguos y es muy elaborada: hay cerca de un centenar de adavus (movimientos básicos que en su conjunto componen una danza), 10 mandalas (posiciones estáticas), y 10 grupos de hastas (gestos con las manos); también existe otro tipo de componentes, como las posiciones de los pies o movimientos de los ojos, entre otros. Así, esta danza es compleja porque incorpora múltiples aspectos corporales que deben ser dominados por el practicante cuando ofrece un recital. La definición de un lenguaje formal facilitará su documentación de manera sistemática, mejorando la comunicación entre sus practicantes y apoyando en su enseñanza. El objetivo de este trabajo fue definir un lenguaje formal básico para la danza Bhāratanātyam que pudiera ser procesado automáticamente por un analizador sintáctico, con la finalidad de facilitar el proceso de su enseñanza-aprendizaje. De todos los elementos de la danza, se seleccionó solo un subconjunto reducido para definir un léxico básico. Se construyó una red de transición aumentada (ATN) para definir el lenguaje formal que incluye nueve clases de símbolos terminales (categorías de palabras) y siete clases de símbolos no terminales o sub-redes. El diseño de lenguajes formales para danzas y su correspondiente gramática ATN, es una herramienta didáctica que auxilia en el proceso de enseñanza-aprendizaje, permitiendo también la documentación de danzas completas que pueden ser compartidas entre los practicantes.
\end{abstract}

Palabras clave: ATN, Danza Bhāratanātyam, Hinduismo, Procesamiento de Lenguaje Natural, Lenguaje de danza Nātya Sūtra, Gramáticas transformacionales.

\section{Augmented Transition Network and Formal Language for Bhāratanātyam Dance}

Abstract. Bhāratanātyam is an ancestral classical Indian dance whose practice has nowadays spread to many countries. The dance is documen- 
ted in ancient texts and is very elaborated: there are around 12 types of items of dances, such as alarippus, padams, jatisvarams, or tilanas, more than a hundred of adavus (basic movements that in conjunction compound a dance), 10 mandalas (static positions), and 10 types of hastas (hand gestures); there are also other components as feet positions, and eyes movements, among others. Thus, this ancestral dance is complex because it incorporates many corporal aspects that should be dominated by the practitioner in order to perform a recital. The definition of a formal language will facilitate the documentation of this dance in a systematic way that will help to improve the teaching-learning process and also the development and communication among the community of its performers. The objective of this work was to define a basic formal language for Bhāratanātyam that could be processed automatically by a syntactic analyzer, in order to facilitate the process of learning and teaching this ancestral Indian dance. From all elements of the dance, a basic subset was selected to define a base lexicon. An augmented transition network (ATN) was built to defined a formal language that includes nine classes of terminal symbols (words categories) and seven classes of non-terminal symbols or sub-networks. The design of formal languages for ancient dances, and its correspondent grammar through ATN, is a didactical tool that facilitates the teaching-learning process, and also the documentation of complete dances that can be shared among the practitioners.

Keywords: ATN, Bhāratanātyam dance, Hinduism, Natural Language Processing, Nātya Sūtra dance language, transformational grammars.

\section{Introducción}

El Bhāratanātyam es una danza ancestral, se le reconoce como una de las ocho danzas clásicas de la India y hoy en día se practica en muchos países [2]. Está documentada en textos ancestrales como el Nātyaśāstra de Bharata-Muni así como en los relieves en piedra de los templos hinduistas en donde se despliegan frases completas de movimientos. El Bhāratanātyam es una danza muy elaborada: existen cerca de 12 tipos de ítems de danzas como alarippus, padams, jatisvarams, o tilanas, más de cien adavus (movimientos básicos que en su conjunto conforman una danza), 10 mandalas (posiciones estáticas), y 10 tipos de hastas (gestos de la mano; Tabla 1); también hay otro tipo de componentes como posiciones de los pies, movimientos de los ojos y del cuello, entre otros [9]. Así, esta danza ancestral es compleja porque incorpora muchos aspectos corporales que el practicante debe dominar al hacer un recital.

De manera típica la danza se enseña mediante ejemplo del profesor, el estudiante memoriza los ejercicios y toma notas con símbolos que inventa, la mayoría de las veces con dibujos caricaturizados del cuerpo humano. Este método tiene la ventaja de que permite al estudiante tomar notas de manera libre, sin embargo, es ineficiente cuando se desea representar danzas de duración larga que se componen 
de varios movimientos. Además, este tipo de notación puede causar confusiones y malinterpretaciones.

El problema de construir un lenguaje para la danza ya se ha plantado con anterioridad. En los inicios del siglo pasado se propuso una notación para danza clásica [10], desafortunadamente el sistema es muy críptico por lo que no ha sido ampliamente usado. En México se propuso otro sistema para danza folklórica $[12,11]$, pero la notación está orientada a representar la coreografía, más que los movimientos del bailarín.

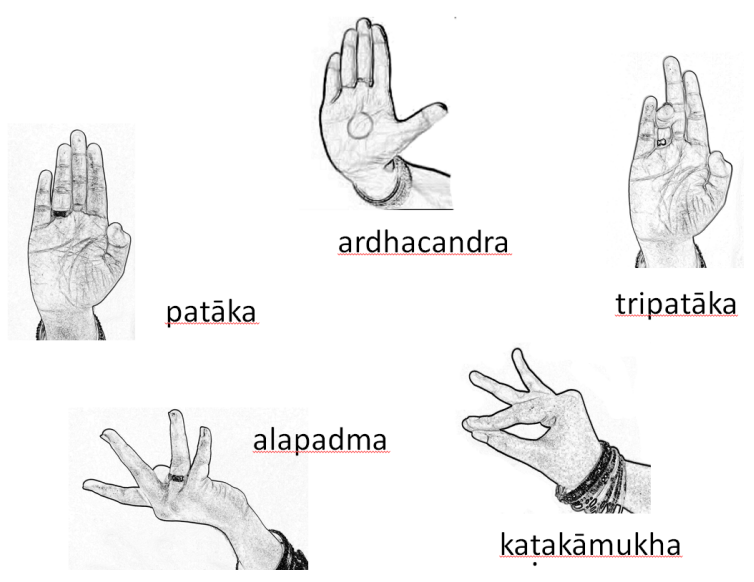

a)

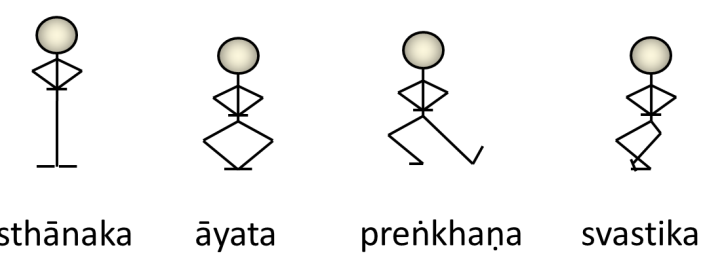

b)

Fig. 1. Algunos ejemplos iconográficos de las posturas de la danza Bhāratanātyam. a) hastas (gestos de las manos); b) mandalas (posiciones de las piernas)

\subsection{Trabajos previos}

Existen trabajos que aplican la informática al análisis de los movimientos y posturas del Bhāratanātyam, uno de ellos [8] propone codificar los pasos de la danza mediante un "vector de posición de danza" que consiste en una lista de números naturales que codifican treinta atributos que corresponden a sies partes del cuerpo, este vector de posición es más bien útil como una 
representación interna más que un lenguaje para el practicante. Otro trabajo [7] que aplica la informática al análisis del Bhāratanāṭam describe un sistema que reconoce gestos de las manos (hastas) mediante análisis de imágenes, y también la representación se orienta a la lectura por una computadora y no por el practicante.

La definición de un lenguaje formal facilitará la documentación de esta danza de manera sistemática y ayudará a mejorar el proceso de enseñanza-aprendizaje y también al desarollo y comunicación entre la comunidad de bailarines. Si el lenguaje formal se puede vincular con herramientas automáticas, entonces la documentación se podrá convertir en formas dinámicas suceptibles de ser analizadas y estudiadas en dispositivos electrónicos.

\subsection{Objetivo}

El objetivo de este trabajo fue definir un lenguaje formal para el Bhāratanātyam que pueda ser procesado automáticamente por un analizador sintáctico, con la finalidad de facilitar la documentación y el proceso de aprendizaje y enseñanza de esta danza ancestral de la India.

\section{Danza y procesamiento de lenguaje natural}

\subsection{Redes de transición aumentada (ATN)}

Una red de transcición aumentada (ATN) es un viejo formalismo para el procesamiento del lenguaje natural (NLP) para analizar la estructura sintáctica de una oración, y fue construido con base en los trabajos seminales de las gramáticas generativas [4]. El formalismo [15,14] representa a una gramática por medio de gráficas compuestas por arcos y nodos, donde los nodos indican cada estado del análisis y los arcos imponen restricciones a la oración que es representada mediante una lista de palabras y símbolos de puntuación. Los arcos se etiquetan con símbolos terminales, i.e. palabras, o con nombres de sub-redes. Por ejemplo, si una oración está formada por una frase nominal seguida de una frase verbal, entonces la gráfica SENTENCE puede ser representada por los arcos: NOUN_PHRASE y VERBAL_PHRASE; a su vez, la gráfica NOUN_PHRASE puede estar conformada por los arcos article y name.

\section{2. $\quad$ El uso de NLP para representación de danzas}

El formalismo ATN deriva directamente de las redes de transición recursivas (RTN), ambas comparten la misma representación, la diferencia principal es que en las ATN se pueden especificar los rasgos y su concordancia, por ejemplo, la concordancia de género, de número y persona, entre otras. Esta característica del ATN puede ser explotada en un lenguaje para la danza porque los movimientos pueden ser exclusivos de partes específicas del cuerpo, i.e., puede existir concordancia entre tipos de movimientos y partes del cuerpo. Otra característica de las danzas, representadas como frases que cumplen restricciones, son los desplazamientos simétricos. 


\section{Método}

La danza Bhāratanātyam tiene muchos elementos que se clasifican y describen sistemáticamente de forma tradicional, por ejemplo, los accesorios, la vestimenta, el maquillaje, o los movimientos del cuello y de los ojos, entre otros, que son considerados parte del lenguaje de la danza [13]. De todos los elementos de la danza, solo se eligió un subconjunto esencial para definir un léxico básico: diez mandalas, dos tipos de adavus (tatta adavus, y națta adavus, 16 en total), un tipo de hastas (28 en total; ver Tabla 1), siete direcciones de movimientos del cuerpo (up, down, front, back, right, left, y sideway), y cuatro posiciones de las piernas (Figura 1).

\subsection{Rasgos y concordancia}

Como muchos de los movimientos se refieren exclusivamente a los pies o a las manos, se incluyó a hand/foot como un rasgo de la red que impone condiciones de concordancia. Por ejemplo, en el léxico se describe para cada palabra, además de la categoría a la que pertence, el rasgo, mediante uno de los tokens hand o foot.

No obstante que la simetría de pies o manos es una característica importante de las secuencias de los movimientos, y por lo tanto es una buena candidata de rasgo, en esta primer versión de la ATN se decidió no incluirla.

\subsection{Adavus como guía para definir el léxico}

La descripción de los movimientos de piernas y manos se encuentra de forma muy detallada en los textos Abhinayadarpana de Nandikervara [6] y Nātyaśāstra de Bharata-Muni [3], en particular, los adavus son combinaciones de posiciones de piernas, formas de pararse, movimientos y hastas (gestos de las manos), y son las secuencias básicas de las que se compone una danza. Así, el léxico básico se creó tomando en cuenta las posiciones y movimientos de dos tipos de adavus: tatța adavus que son pasos básicos que involucran movimientos sólo con las piernas, y los natta adavus que además involucran movimientos de brazos, incluyendo gestos con las manos.

\subsection{Representación en lenguaje Prolog del analizador ATN}

El léxico básico se agrupó en nueve categorías de palabras (Tabla 2) que se seleccionaron con base en dos criterios: 1) las palabras con las que se podrían describir los movimientos y partes del cuerpo involucrados en los tatta adavus y natta adavus, y 2) las palabras que representan la lista de posiciones y movimientos básicos: hastas o gestos con las manos, y mandalas o posiciones de los pies (Figura 1).

El predicado Prolog para representar las palabras es word/3:

word (Word_category, Word, Feature $=\langle$ foot/hand/_>). 
donde Word_category es la categoría de Word, v.gr. una preposición, es decir, de la categoría preposition (at, to) o action_word (hit, rotate) (Tabla 2); y Feature indica si la palabra Word tiene restricción de concordancia (hand/foot) o no (_). Sólo se consideró un rasgo para concordancia, que verifica que el movimiento sea congruente con la parte del cuerpo, por ejemplo, la posición ardhacandra es una hasta, i.e., un gesto de mano, entonces, la expresión right hand ardhacandra es válida, mientras right leg ardhacandra es inválida; los hechos Prolog usados para representar a las palabras involucradas son:

word (body_side, right,__).

word (body_part_word, hand, hand).

word (position, ardhacandra, hand).

word(body_part_word, leg, foot).

Todas las asamyuta hastas (Tabla 1) tienen asociada la etiqueta hand, y se representan con hechos del siguiente tipo word(position, ardhacandra, hand). Algunos ejemplos de palabras que no tienen concordancia son:

word (preposition, at, _).

word (preposition, to, _).

word (body_part_word, head, _).

word (body_part_word, trunk, _).

Construimos el código del analizador ATN en lenguaje Prolog tomando como base el código del libro de texto de procesamiento de lenguaje natural de Gazdar y Mellish [5]. En el lenguaje Prolog los hechos se pueden representar directamente, y la máquina de backtrack automático facilita la programación del formalismo ATN, que básicamente consiste en la búsqueda de un camino en las redes, y que representa la solución del análisis sintáctico. Las redes se representan con el predicado arc/4:

arc(Node_from, Node_to, Word_or_Network, Network_of_this_arc)

donde Node_from y Node_to son el inicio y fin del arco arc, respectivamente, Word_or_Network es una palabra o la etiqueta de una red (Network), y Network_of_this_arc es el nombre de la red a la que pertenece el arco arc.

\section{Resultados}

\subsection{El lenguaje formal propuesto: léxico y notación}

El lenguaje formal incluye nueve clases de símbolos terminales (categorías de palabras; Tabla 2). Cada oración se forma por una secuencia de palabras, por ejemplo, las palabras de la oración:

stance mandala sthanaka; arms back hasta ardhacadra look at front; end_stance. 


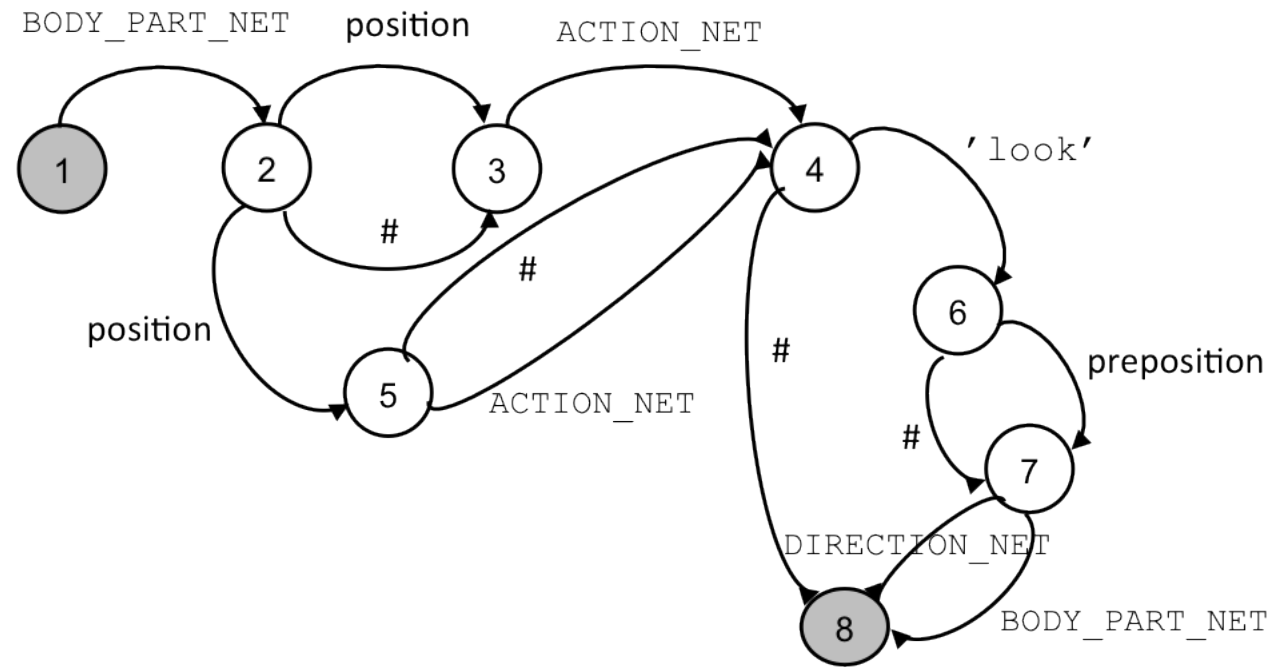

Fig. 2. La red BODY_PART_MOVEMENT_NET del analizador ATN. Analiza oraciones del Bhāratanātyam que involucran movimientos de piernas, manos, tronco, entre otros. Los nodos 1 y 8 son el inicial y el final de la red, respectivamente. El símbolo \# denota un salto, i.e., el análisis continúa en el siguiente nodo del arco correspondiente

tienen las siguientes categorías:

stance.- constante que identifica una posición estática

mandala.- constante que identifica posiciones de las piernas

sthanaka.- position

arms.- - body_part_word

back.- direction_word

hasta.- body_part_word

ardhacadra.- position

look.- constante que identifica que lo que sigue es un drști bheda (movimientos de los ojos)

at.- prepositon

front.- direction

end_stance.- constante que identifica el fin de la oración

Esta oración se puede entender mejor si se observa la Figura 1, y corresponde a los primeros elementos de la Figura 3a y 3b. La estructura sintáctica de la oración se incluye en la Figura 4.

\subsection{La gramática ATN propuesta para la danza Bhāratanātyam}

La gramática del lenguaje Nātya Sūtra contiene siete clases de símbolos no terminales, así, la ATN se compone de siete sub-redes (Tabla 3), que son: 
PARTIAL_PHRASE_NET, BODY_PART_MOVEMENT_NET, STANCE_NET, BODY_PART_NET, ACTION_NET, DIRECTION_NET, y HIT_SEQUENCE_NET (Figura 2).

La red que procesa a las frases de partes del cuerpo es BODY_PART_NET, que consiste de tres nodos y tres arcos:

$\operatorname{arc}(1,2$, body_side, body_part).

$\operatorname{arc}(1,2$, '\#', body_part).

$\operatorname{arc}(2,3$, body_part_word, body_part).

la red comienza en el nodo 1 , y termina en el nodo 3 :

begin (1, body_part).

end (3, body_part).

las dos plabaras body_side registradas en el léxico son:

word (body_side, right,_).

word (body_side, left,_).

y las palabras body_part_word registradas en el léxico son:

word (body_part_word, body, _).

word (body_part_word, trunk, _).

word (body_part_word, leg, foot).

word(body_part_word, legs, foot).

word(body_part_word, foot, foot).

word(body_part_word, feet, foot).

word (body_part_word, hand, hand).

word (body_part_word, hasta, hand).

word(body_part_word, hands, hand).

word (body_part_word, arm, hand).

word (body_part_word, arms, hand).

word (body_part_word, head, _).

word (body_part_word, neck, _).

word (body_part_word, mandala, foot).

En la Figura 3 se muestra el tatta adavu 5 en el lenguaje Nātya Sūtra y la estructura sintáctica de sus frases. Como un ejemplo, para la siguiente frase:

stance mandala sthanaka; arms back hasta ardhacandra look at front; end_stance.

la salida del procesamiento por la ATN, que es la estructura sintáctica propuesta, se muestra en la primer columna de la Figura 4.

\section{Discusión}

El lenguaje Nātya Sūtra para Bhāratanātyam es una herramienta para la enseñanza y el aprendizaje de esta danza ancestral, además, mediante este lenguaje se pueden documentar danzas completas. La gramática propuesta incluye 
un léxico base que puede ampliarse para incorporar más elementos como son movimientos del cuello y ojos, entre otros. El manejo de rasgos se ejemplifica con solo una característica, pero se podrán incorporar más rasgos, como por ejemplo la simetría: proponemos que una COMPLETE_PHRASE puede descomponerse en dos PARTIAL_PHRASE que se complementen una a otra en simetría, derecha e izquierda, y que una DANCE se forme de un conjunto de PARTIAL_PHRASE, y que contenga cuando menos una COMPLETE_PHRASE, es decir, que contenga al menos una secuencia derecha que se repita en su análoga izquierda.

Con el lenguaje formal propuesto se pueden representar danzas ancestrales y puede usarse para soportar las dos posiciones dialécticas: ortodoxia e innovación. Para los innovadores, los beneficios de usar gramáticas ATN para danzas ancestrales se pueden visualizar fácilmente, por ejemplo, se podrían crear nuevas secuencias, incluso danzas completas que siguieran las reglas impuestas en la gramática; por otra parte, para los ortodoxos, nos gustaría considerar el punto de vista de la gran bailarina Balasaraswati quien dijo que "las más grandes autoridades de la danza han reconocido definitivamente que es la ortodoxia de la disciplina tradicional la que da la más amplia libertad para la creatividad individual del bailarín" [1]. Nosotros comulgamos con esa idea, y proponemos al formalismo ATN y al lenguaje Nātya Sūtra como una herramienta para documentar y preservar al Bhāratanāṭam.

\section{Trabajo futuro}

Hay diversos elementos de la danza aún no incorporados en el lenguaje que requieren su adaptación y nomenclatura, y quizá la definición de nuevas categorías de palabras y subredes gramaticales. La capacidad de los ATN para procesar rasgos y que en esta primera versión del lenguaje se ejemplifica con la verificación de concordancia entre manos y pies, puede ser mejor aprovechada incorporando restricciones adicionales, como por ejemplo la simetría izquiera/derecha en la secuencia de los movimientos, característica de esta danza.

\section{Conclusiones}

El diseño de lenguajes formales para danzas ancestrales y sus correspondientes gramáticas mediante redes de transición aumentada, constituyen herramientas que pueden facilitar el proceso de enseñanza-aprendizaje, así como la documentación de danzas completas que pueden ser compartidas entre sus practicantes.

El lenguaje Nātya Sūtra descrito en este trabajo puede ser de utilidad a los practicantes de la danza Bhāratanātyam, tanto maestros como alumnos. Los nombres cortos para las asamyuta hastas, o gestos de una mano, y las categorías de palabras del lenguaje propuesto constituyen herramientas útiles al tomar notas ante una presentación, permitiendo el registro más rápido que de la manera tradicional mediante caricaturas del cuerpo humano. 
Agradecimientos Nuestro querido upādhyāyah, el Dr. Roberto García Fernández de Sánscrito en México, revisó la transliteración de las palabras en lengua sánscrita. Gabriela Murguía-Romero revisó el texto de una versión preliminar del manuscrito. RRC y MMR agradecen a Patricia Torres (ICCR Gurudev Tagore México, Gobierno de la India), Fabiola Ocón (Danzas de la India) y Julieta Solís (Opus Uno), y MMR a Sagrario T. Gómez y Natalia Cárdenas (Dirección General de Danza, UNAM), por sus enseñanzas sobre Bhāratanātyam.

\section{Referencias}

1. Balasaraswati, T.: On Bharata Natyam. Dance Chronicle 2, 106-116 (1978)

2. Balasubramaniam, C.: Language of the soul. Published by Public Diplomacy Division, New Delhi, for the Ministry of Externals Affairs, India. November-December 26, 58-63 (2012)

3. Bharata-Muni: The Nātyaśāstra. Transalted into English by Manmohan Ghosh. Bibliotecha Indica. Iissue no. 1559, work no.272. Asiatic Society of Bengal. Calcuta (1951)

4. Chomsky, N.: On certain formal properties of grammars. Information and Control 2, 137-167 (1959)

5. Gazdar, G., Mellish, Ch.S.: Natural language processing in Prolog: an introduction to computational linguistics. Addison-Wesley (1989)

6. Ghosh, M.E.: Nandikesvaras Abhinayadarpanam: a manual of gesture and posture used in Hindu dance and drama. KL Mukhopadhyay. Calcuta (1957)

7. Hariharan, D., Acharya, T., Mitra, S.: Recognizing hand gestures of a dancer. In: Kuznetsov, S. O. (ed), PReMI 2011, LNCS 6744. Springer-Verlag Berlin Heidelberg. pp. 186-192 (2011)

8. Jadhav, S., Joshi, M., Pawarm, J.: Modeling BharataNatyam dance steps: Art to smart. In: Proceedings of the CUBE International Information Technology Conference 2012. pp. 320-325. Pune, Maharashtra, India (September 2012)

9. Krishna Rao, U., U.K. Chandrabhaga Devi : A Panorama of Indian Dances. Sri Satguru Publications (1993)

10. von Laban, R.: Coreografía. Primer cuaderno. Eugen Diederichs, Jena, Germany (1926). Translated by Carla Doniz Geist, Instiuto Nacional de Bellas Artes y Literatura, México. 141pp (2013)

11. Nuñez Mesta, M.A., Reyes Gómez, L., de Anda Esquivel, F.: Bailes del folklor mexicano, 5 vols. Trillas, México (2001)

12. Nuñez Mesta, M.A., Reyes Gómez, L., de Anda Esquivel, F.: Bailes del folklor mexicano: metodología de la enseñanza mediante el sistema ACADEDA. Trillas, México (2001)

13. Venkataram, L.: Harya in classical dances. India Perspectives. Published by Public Diplomacy Division, New Delhi, for the Ministry of Externals Affairs, India. November-December 24, 67-79 (2010)

14. Winograd, T.: Language as a Cognitive Process. Vol. 1. Syntax. Addison-Wesley (1983)

15. Woods, W.A.: Transition network grammars for natural language analysis. Commun. ACM 13, 591-606 (1970) 
Tabla 1. Asamyuta hastas (gestos de una mano) usados en la danza Bhāratanātyam y sus correspondientes símbolos propuestos en el lenguaje formal Nātya Sūtra. Proponemos que los símbolos cortos se formen concatendando la letra ' $\mathrm{H}$ ' a las cuatro primeras letras del nombre sánscrito, excepto para la hasta hamsapakṣa, para diferenciarla de hamsasya

\begin{tabular}{|c|c|c|c|c|c|}
\hline & $\begin{array}{l}\text { Nombre en } \\
\text { sánscrito }\end{array}$ & $\begin{array}{l}\text { Nombre } \\
\text { corto }\end{array}$ & $\begin{array}{l}\text { Símbolo de } \\
\text { átomo Prolog }\end{array}$ & $\begin{array}{l}\text { Símbolo } \\
\text { Prolog }\end{array}$ & $\begin{array}{l}\text { corto Símbolo Prolog } \\
\text { alterno }\end{array}$ \\
\hline$\overline{1}$ & patāka & Hpata & pataka & hpata & h01 \\
\hline 2 & tripatāaka & Htrip & tripataka & htrip & h02 \\
\hline 3 & ardhapatāka & Hardh & ardhapataka & hardh & h03 \\
\hline 4 & kartarīmukha & Hkart & kartarimukha & hkart & h04 \\
\hline 5 & mayūra & Hmayu & mayura & hmayu & h05 \\
\hline 6 & ardhacandra & Hardh & ardhacandra & hardh & h06 \\
\hline 7 & arāla & Haral & arala & haral & h07 \\
\hline 8 & śukatunda & Hsuka & sukatunda & hsuka & h08 \\
\hline 9 & mușți & Hmust & musti & hmust & h09 \\
\hline & śikhara & Hsikh & sikhara & hsikh & h10 \\
\hline & kapittha & Hkapi & kapittha & hkapi & h11 \\
\hline & 2 kațakāmukha & Hkata & katakamukha & hkata & h12 \\
\hline & sūcī & Hsuci & suci & hsuci & h13 \\
\hline & 4 candrakalā & Hcand & candrakala & hcand & h14 \\
\hline & 5 padmakośa & Hpadm & padmakosa & hpadm & h15 \\
\hline & sarpaśiras & Hsarp & sarpasiras & hsarp & h16 \\
\hline & 7 mṛgaśīisṣa & Hmrga & mrgasirsa & hmrga & h17 \\
\hline & s simphamukha & Hsimh & simhamukha & hsimh & h18 \\
\hline & kan்gula & Hkang & kangula & hkang & h19 \\
\hline & alapadma & Halap & alapadma & halap & h20 \\
\hline & catura & Hcatu & catura & hcatu & h21 \\
\hline & bhramara & Hbhra & bhramara & hbhra & $\mathrm{h} 22$ \\
\hline & 3 hampasya & Hhams & hamsasya & hhams & h23 \\
\hline & 4 haṃsapakṣa & Hhamp & hamsapaksa & hhamp & h24 \\
\hline & sandamśa & Hsand & sandamsa & hsand & h25 \\
\hline & mukula & Hmuku & mukula & hmuku & h26 \\
\hline & 7 tāmracūḍa & Htamr & tamracuḍa & htamr & h27 \\
\hline & triśūla & Htris & trisula & htris & h28 \\
\hline
\end{tabular}


Tabla 2. Categorías de palabras definidas en el lenguaje formal Nātya Sūtra

\begin{tabular}{|c|c|}
\hline $\begin{array}{l}\text { Categoría } \\
\text { de palabra }\end{array}$ & Descripción [Ejemplos de palabras] \\
\hline \multicolumn{2}{|c|}{$\begin{array}{l}\text { body_part_word La parte del cuerpo para la que se describe un movimiento } \\
\text { [foot }],[\text { hand }],[\text { head }],[\text { leg }],[\text { trunk }], \ldots\end{array}$} \\
\hline body_side & $\begin{array}{l}\text { Especifica si se refiere a la parte derecha o izquierda [left], [right], } \\
\text { por ejemplo, usadas en las expresiones [left hand], o [right leg]. }\end{array}$ \\
\hline action_word & La acción que se realiza por alguna parte del cuerpo [hit], [rotate]. \\
\hline preposition & $\begin{array}{l}\text { Preposición opcional que se puede usar antes de una dirección [at, to], } \\
\text { por ejemplo, en expresiones como [to left], [at front], ... }\end{array}$ \\
\hline position & $\begin{array}{l}\text { Posiciones de las piernas (mandalas) [sthanaka], [svastika], ..., } \\
\text { posiciones estandarizadas de piernas, brazos y manos, } \\
\text { y hastas }[\text { pataka], [ardhacandra], ... }\end{array}$ \\
\hline direc & Direcciones básicas en el espacio [down], [up], [left], [right], .. \\
\hline adavu & $\begin{array}{l}\text { Secuencias básicas estandarizadas de movimientos } \\
\text { [tatta_adavu_1], [tatta_adavu_2], ... [natta_adavu_1] }\end{array}$ \\
\hline & Números naturales $[1,2,3,4,5,6,7,8,9, \ldots]$ \\
\hline separator & Separador opcional de secuencias de movimientos $[;]$ \\
\hline
\end{tabular}

Tabla 3. Redes definidas en el lenguaje formal Nātya Sūtra

\begin{tabular}{ll}
\hline Red & Descripción \{ejemplo de frase reconocida\} \\
\hline partial_phrase_net & Frases o secuencias de una o más body_part_movement o \\
& stance (ver red body_part_movement_net y stance_net): \\
& [stance mandala sthanaka arms back hasta ardhacandra look at \\
& front end_stance; stance mandala aayata arms back hasta \\
& ardhacandra end_stance.] \\
body_part_movement_net & Realiza el "parsing" de las frases acerca del movimiento de \\
& una parte específica del cuerpo, como feet, trunk, arms, hands: \\
& [mandala aayata right foot hit.] \\
stance_net & Muy similar a body_part_movement_net, usada para describir po- \\
& siciones no dinámicas del cuerpo, i.e., la posición de partes del \\
& cuerpo en un instante, y no en una secuencia a través del tiempo: \\
& [stance mandala sthanaka arms back hasta ardhacandra look at \\
& front end_stance.] \\
body_part_net & Identifica partes del cuerpo: [right foot.], [trunk.], [legs.], \\
& [left hand.], [head.] \\
action_net & Identifica acciones que principalmente se realizan con pies y manos: \\
direction_net & [rotate.], [hit.] \\
& Identifica las direcciones en el espacio de los movimientos: [right.], \\
hit_sequence_net & [down front.] \\
& Identifica acciones de golpeteo con el pie: [left hit 2 speed 1 \\
& pause hit 3 speed 2.]
\end{tabular}



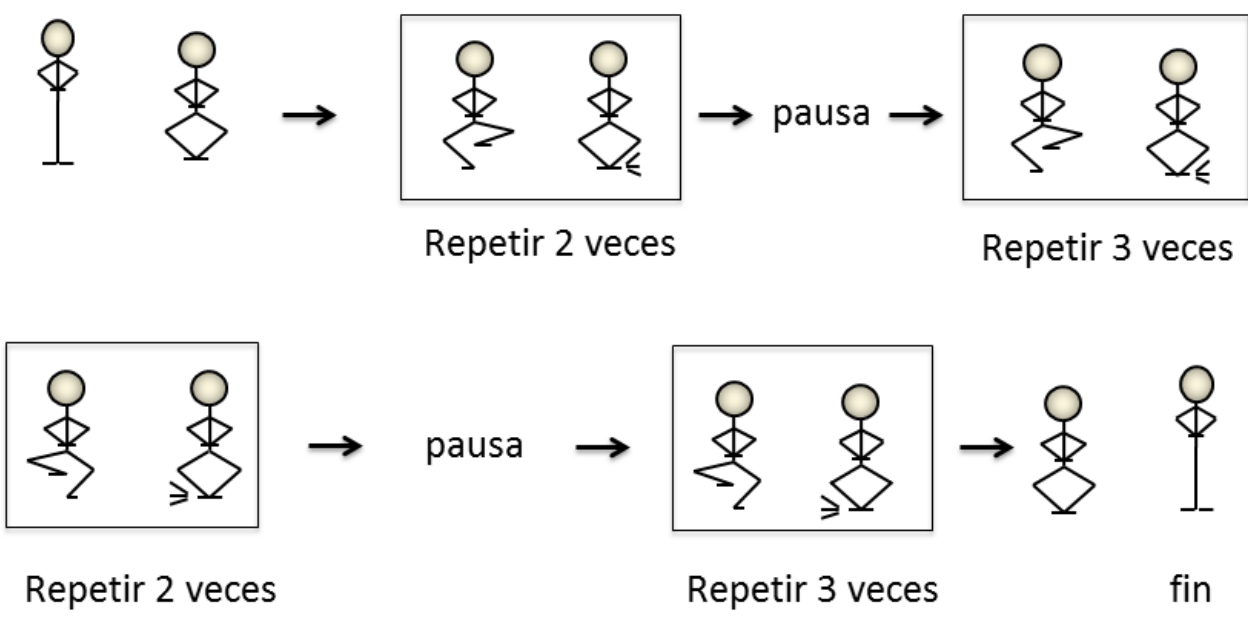

a)

\section{$\%$ Preparacion}

stance mandala sthanaka; arms back hasta ardhacandra look at front; end_stance. stance mandala aayata; arms back; hasta ardhacandra; end_stance.

$\%$ tatta adavu 5

mandala aayata right hit 2 speed 1 pause hit 3 speed 2.

mandala aayata left hit 2 speed 1 pause hit 3 speed 2.

$\%$ Final

stance mandala sthanaka; arms back; hasta ardhacandra look to front; end_stance.

b)

$?-\operatorname{atn}([$

$\%$ PREPARACION

stance, mandala, sthanaka, arms, back, hasta, ardhacandra, look, at, front, end_stance, stance mandala, aayata, arms, back, hasta, ardhacandra, end_stance, $\%$ TATTA ADAVU 5

mandala, aayata, right, hit, 2 , speed, 1, pause, hit, 3, speed, 2, mandala, aayata, left, hit, 2, speed, 1, pause, hit, 3, speed, 2, $\%$ FINAL

stance, mandala, sthanaka, arms, back, hasta, ardhacandra, look, to, front, end_stance ], Parse).

c)

Fig. 3. Ejemplo de una oración del lenguaje Nātya Sūtra. a) Secuencia iconográfica del tatța aḍavu 5; b) La oración del tatța aḍavu 5 escrita en lenguaje Nātya Sūtra; c) Representación Prolog de la oración en a) y b) aceptada como entrada del ATN 


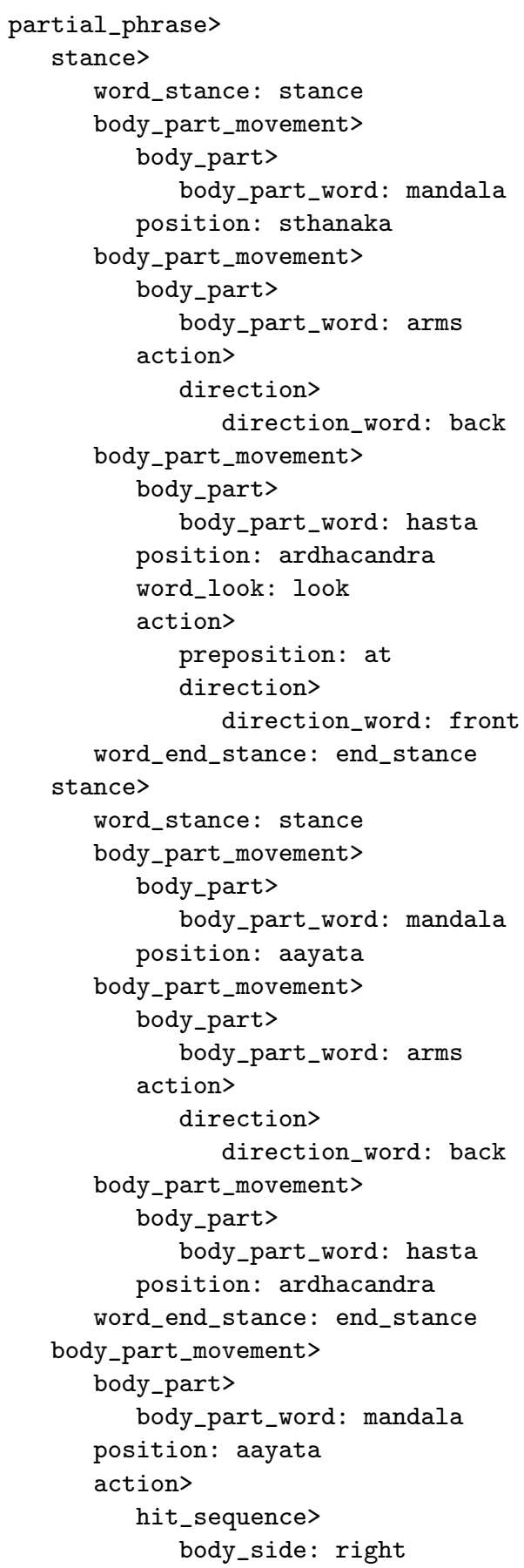

Fig. 4. Estructura sintáctica de la oración de la Figura 3 producida por el analizador ATN 